\title{
Féminisme d'État, législation et mouvements sociaux en Turquie et en Grèce
}

State Feminism, Legislation and Social Movements in Turkey and Greece

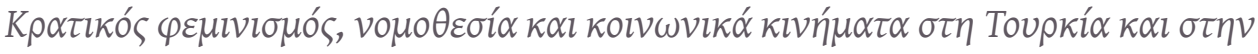

$E \lambda \lambda \alpha ́ \delta \alpha$

\section{Katerina Seraïdari}

\section{OpenEdition}

\section{Journals}

Édition électronique

URL : https://journals.openedition.org/ceb/9919

DOI : 10.4000/ceb.9919

ISSN : 2261-4184

Éditeur

INALCO

Édition imprimée

ISBN : 978-2-85831-239-9

ISSN : 0290-7402

Référence électronique

Katerina Seraïdari, «Féminisme d'État, législation et mouvements sociaux en Turquie et en Grèce », Cahiers balkaniques [En ligne], 44 | 2016, mis en ligne le 04 janvier 2018, consulté le 07 juillet 2021.

URL : http://journals.openedition.org/ceb/9919; DOI : https://doi.org/10.4000/ceb.9919

Ce document a été généré automatiquement le 7 juillet 2021.

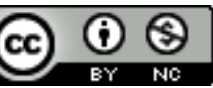

Cahiers balkaniques est mis à disposition selon les termes de la Licence Creative Commons Attribution - Pas d'Utilisation Commerciale 4.0 International. 


\title{
Féminisme d'État, législation et mouvements sociaux en Turquie et en Grèce
}

\author{
State Feminism, Legislation and Social Movements in Turkey and Greece

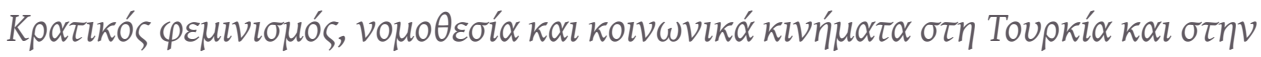 \\ $E \lambda \lambda \alpha ́ \delta \alpha$
}

Katerina Seraïdari

1 Le féminisme d'État est défini ici comme l'action de l'État en faveur des femmes. Il est envisagé comme un vecteur de revendications formulées par des féministes et d'autres mouvements contestataires, préexistants ou parallèles; autrement dit, il résulte des interactions entre les sphères du pouvoir et une base dont l'idéologie et l'origine sociale diffèrent selon les périodes. Par exemple, pour la Turquie, elle était constituée d'une élite bourgeoise à l'époque des réformes kémalistes, tandis qu'elle était plutôt liée aux mouvements de gauche dans les années $1980^{1}$. Par conséquent, le schéma esquissé en rapport avec le féminisme d'État n'est pas forcément vertical (du haut vers le bas), dans la mesure où il reflète également les négociations et les rapports de pouvoir (horizontaux) entre différents acteurs. Toutefois, par ses initiatives, le pouvoir central finit par pousser les mouvements de femmes vers la dé-radicalisation et la bureaucratisation. Ainsi, les associations féministes, auxquelles des subventions sont octroyées, deviennent, dans une large mesure, dépendantes de l'État ${ }^{2}$.

Une définition interactive du féminisme d'État présuppose l'émancipation active des femmes, qui ne sont pas de sujets passifs auxquels des droits sont octroyés par un leader charismatique dont l'action fait avancer la société tout entière. Elle va donc à l'encontre d'une notion de leadership qui infantilise les membres d'une société. En effet, le féminisme d'État ne se développe pas sur une tabula rasa, mais constitue la suite d'une histoire entamée par des acteurs initialement sans accès au pouvoir : avant de devenir une affaire d'État, le féminisme connaît une phase pré-étatique. 
3 Même si c'est le degré de mobilisation qui fait la différence entre les « droits acquis » et les «droits octroyés », aucune réforme ne peut être réalisée, y compris dans un régime autoritaire comme celui que Mustafa Kemal a instauré en Turquie, sans avoir des appuis parmi certaines couches de la population et sans des interactions permanentes entre le pouvoir central et cette base. C'est parce qu'une partie considérable de la bourgeoisie turque était déjà occidentalisée que le projet modernisateur de Kemal a pu voir le jour. Cependant, cette modernité avait surtout imprégné le mode de vie urbain, et seuls $20 \%$ de la population vivaient à l'époque en ville.

4 Les réformes de Mustafa Kemal Atatürk ne doivent pas non plus occulter le rôle qu'un mouvement féministe préexistant avait joué. Dans les dernières décennies de l'Empire ottoman et pendant la première période du régime kémaliste, des féministes turques étaient actives, tant au niveau national qu'international ; certaines assistaient régulièrement à des congrès internationaux entre 1910 et $1930^{3}$. En avril 1935, l'Union des femmes turques a même organisé et hébergé le douzième Congrès suffragiste international de l'Association internationale pour le suffrage des femmes à Istanbul.

5 Même si Atatürk est présenté comme «le sauveur » et le «libérateur " des femmes turques ${ }^{4}$, il serait inexact de parler d'un « féminisme d'homme " puisque l'organisation et l'action des féministes turques avaient précédé les réformes étatiques entreprises par le régime kémaliste ${ }^{5}$. Il est pourtant indéniable que le féminisme d'État en Turquie a fonctionné comme un outil de contrôle social. L'Union des femmes turques a été dissoute en 1935, juste après le Congrès suffragiste international, parce que, selon l'annonce officielle, le régime ayant aboli les différences de sexe et de classes, ce type de mobilisation s'avérait dorénavant inutile.

6 Dans le cas de la Turquie, le féminisme d'État a également été le résultat de conditions socio-économiques favorables : d'un côté, la Première Guerre mondiale et la Guerre de libération (contre l'armée grecque) ont donné un nouveau statut aux femmes qui ont remplacé, aux différents postes de travail, les hommes qui partaient se battre; de l'autre côté, les femmes ont pu occuper des places qui leur étaient jadis inaccessibles, parce que le départ des élites chrétiennes a laissé un grand vide qu'il fallait rapidement combler. Dans ce cadre, l'amélioration de la condition féminine et l'accès à l'éducation et au monde du travail sont liés à un projet basé sur l'idée de libération nationale, ainsi qu'au passage d'un Empire multiculturel à l'État-nation.

7 Le but de cet article est d'analyser les variations du fonctionnement du féminisme d'État et son rapport avec le droit qui constitue, comme nous le verrons, une construction sociale et politique malléable. Dans un premier temps, je présenterai les réformes entreprises en Turquie kémaliste. Puis, j'examinerai comment le pouvoir central dans deux pays voisins et " frères ennemis ", la Turquie et la Grèce, a utilisé le féminisme comme outil de modernisation dans les années 1920-1930. Dans un deuxième temps, j'analyserai l'impact de la " seconde vague » du féminisme ${ }^{6}$ dans ces deux pays depuis les années 1980 et ses rapports avec l'État et sa politique. Enfin, je présenterai l'ONG Winpeace, qui rassemble depuis 1996 des féministes grecques et turques et dont l'action révèle comment les mouvements sociaux et le féminisme d'État interagissent actuellement. 


\section{Les réformes en Turquie} marquent «le passage d'un mode de vie "homosocial" à un mode de vie "hétérosocial" " et le passage de la ségrégation des sexes à la mixité7. Cette loi reconnaît le droit à l'enseignement pour les deux sexes et conduit à la création des écoles mixtes ; les écoles religieuses et l'enseignement religieux sont alors supprimés. En 1926, la loi islamique est abolie et remplacée par un code civil calqué sur le Code civil suisse. Ce corpus législatif joint donc les traditions culturelles de deux pays géographiquement et culturellement lointains.

9 Jacques Lafon considère que le code suisse était simple et moderne à la fois, contrairement au code français (jugé désuet) et au code allemand (perçu comme trop compliqué) $^{8}$. Lafon évoque la présence déterminante des "Lausannois», des intellectuels turcs francophones qui avaient fait des études de droit en Suisse : parmi eux figurait Mahmut Esat qui est devenu ministre de la Justice ${ }^{9}$. Mais Lafon montre également que si le code français n'est pas choisi, c'est parce que, «[m]algré sa francophilie, Atatürk se devait de marquer une certaine réserve à l'égard d'une puissance d'occupation ${ }^{10} »$. Sirin Tekeli avance une hypothèse similaire qui révèle à quel point le féminisme d'État est lié à des enjeux politiques qui dépassent la sphère nationale et qui nous font entrer dans la sphère des relations internationales. Selon elle, ce choix

semble avoir été motivé par le fait que la Suisse, pays neutre et ami, se trouvait dans une position politique plus acceptable que les autres pays occidentaux qui avaient été les ennemis de la Turquie pendant la Première Guerre mondiale et la Guerre d'Indépendance ${ }^{11}$.

Birol Baskan lie le nouveau code au traité de Lausanne (1923) qui définit les frontières du nouvel État turc et qui fixe le statut juridique d'environ 2 millions de personnes, dont la grande majorité participe à l'échange de populations entre la Grèce et la Turquie $^{12}$. Selon Baskan, étant obligé d'adopter un système de lois occidentales pour ses minorités non échangées, le régime kémaliste a préféré instaurer un système légal unifié à l'intérieur de son territoire. Mais, au moment où l'article 10 de la Constitution turque établit l'égalité légale entre les deux sexes, les populations turques non échangées en Thrace (Grèce) restent soumises à la loi religieuse, selon les conditions posées par le traité de Lausanne, et ce jusqu'à aujourd'hui.

11 Le nouveau Code civil instaure la monogamie et interdit la polygamie ${ }^{13}$. Il établit des droits égaux entre femme et homme face au divorce, ainsi que le partage de l'autorité parentale, l'égalité dans l'héritage et la succession, l'égalité du statut de témoin en justice et l'obligation de célébrer le mariage en présence de la mariée. Il fixe l'âge légal du mariage à 15 ans pour la femme et à 17 ans pour l'homme. Sur ce dernier point, le Code civil turc se différencie du Code civil suisse et diminue la limite d'âge requis pour le mariage ${ }^{14}$ : cela constitue un exemple de l'adaptation d'un code importé à la réalité sociale turque.

12 Cependant, le nouveau Code civil promulgue une égalité des sexes limitée : la femme n'est pas considérée comme une partenaire égale de l'homme et la question de sa domination ne se pose même pas. L'homme reste le chef de famille (article 152) et la femme, sa subordonnée: c'est l'homme qui doit subvenir aux besoins de la famille, 
tandis que la femme est responsable des activités ménagères (article 153). La femme doit habiter au domicile choisi par l'époux d'après l'article 21 (ce qui constitue une limitation de sa liberté de mouvement). Elle ne peut pas non plus garder son nom de jeune fille (ce qui correspond à une perte d'identité). Le 21 juin 1931 est promulguée la Loi sur les noms de famille qui oblige tous les citoyens turcs à choisir dans un délai déterminé un nom de famille, institution qui n'existait pas juridiquement en Turquie et qui finira par transformer Mustafa Kemal en Mustafa Kemal Atatürk. Ce qui signifie qu'avant que tous les Turcs aient un nom de famille enregistré par les services de l'État, le Code civil (du fait de son origine suisse) posait déjà la question du nom de jeune fille : cela révèle le décalage que peut créer la «transplantation juridique » d'un code civil étranger.

13 Le 3 avril 1930, les femmes turques obtiennent le droit de vote et d'éligibilité aux élections municipales. Sirin Tekeli associe de nouveau à la politique extérieure l'élargissement au niveau national des droits électoraux des femmes en 1934 :

Le fait que la République était un régime à parti unique et de nature autoritaire lui attirait des critiques, de l'intérieur comme de l'extérieur. [...] C'est en grande partie pour désamorcer ces critiques, notamment celles qui venaient de l'extérieur, pour montrer au monde que le régime turc était différent du fascisme, alors croissant, qu'Atatürk a saisi l'occasion d'accorder aux femmes la citoyenneté en 1934, soit un an après l'arrivée au pouvoir de Hitler en Allemagne ${ }^{15}$.

Dans ce cas, le féminisme d'État permet au pouvoir central de se doter d'une image positive au niveau international, d'autant plus que le fascisme (dont le régime kémaliste semble vouloir se différencier) a prôné le retour de la femme au foyer. Il faut aussi rappeler que les femmes turques n'ont accédé au suffrage et à l'éligibilité que quelques années après les hommes ${ }^{16}$. Une fois donné le droit de vote aux femmes, l'âge légal des électeurs a augmenté : de 18 ans (comme cela avait été fixé en 1923) à 22 ans pour les élections «mixtes» de $1935^{17}$. L'entrée des femmes dans la sphère politique a donc fait reculer la limite de l'âge requis comme signe de maturité du citoyen-électeur.

Ces réformes ont significativement changé la vie des populations urbaines et de la bourgeoisie turque; en revanche, les populations rurales ont été très peu touchées. Même si le mariage civil devient obligatoire et le mariage religieux facultatif, le mariage uniquement religieux reste très populaire dans les campagnes, et seule la bénédiction de l'officier du culte musulman est recherchée. Des campagnes administratives en 1933, en 1945 et en 1950 auront pour but d'officialiser le statut de ces couples religieusement mariés, mais non inscrits sur les registres de l'état-civil et de leurs enfants qui n'étaient jusque-là pas reconnus comme légitimes par la

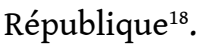

Dans plusieurs cas cependant, les droits constitutionnels n'ont jamais été respectés. Ainsi, un grand nombre de femmes turques n'ont jamais été scolarisées, ce qui va à l'encontre du droit constitutionnel à l'éducation ${ }^{19}$. Peut-on parler dès lors d'un échec des stratégies juridiques? Ce bilan mitigé nous pousse surtout à distinguer les lois de leur application ${ }^{20}$ : les femmes n'ont pas souvent recours aux possibilités que leur offre le cadre légal pour se défendre, car elles ignorent tout simplement l'existence des lois en leur faveur. Ainsi, concernant l'héritage, au lieu d'avoir des droits égaux comme le Code civil l'exige, les femmes turques continuaient de recevoir une petite partie de l'héritage familial ou en étaient exclues, les familles voulant éviter l'éparpillement de la propriété foncière ${ }^{21}$. De ce point de vue, la réforme juridique n'est qu'une première étape, puisque ce qui compte est l'application et la connaissance du cadre légal et des 
droits que celui-ci donne, ce qui renvoie à la distinction entre égalité formelle et substantielle.

Une des modifications apportées au Code civil suisse concernait le régime matrimonial. Si dans le code suisse le régime ordinaire est la communauté de biens, dans le Code civil turc, cette place est donnée au régime de propriété séparée des époux: "C'est une survivance de l'ancienne loi religieuse qui reconnaissait le droit de la femme à posséder ses propres biens ${ }^{22}$.» Pour certaines féministes turques comme Sirin Tekeli, cela n'est pas problématique: une loi islamique ne doit pas être rejetée "si elle comporte des éléments qu'on peut utiliser à l'avantage des femmes ${ }^{23}$ ». Jenny White va encore plus loin quand elle soutient que certains articles du nouveau Code civil n'étaient pas aussi favorables pour la femme que la loi religieuse : alors que la loi islamique prévoyait une somme d'argent qui serait donnée à la femme lors du mariage et du divorce, le Code civil ne lui donnait pas de telles garanties ${ }^{24}$. Selon la loi islamique, cette somme appartenait à la femme, et pas à son mari ni à ses parents, et elle était donc considérée comme une forme de richesse féminine qui l'assurait en cas de veuvage, abandon ou divorce.

Le Code civil turc, qui s'est construit à partir du Code civil suisse (dont proviennent $65 \%$ des textes), constitue en fait un mélange de traditions, où l'on retrouve même des échos de la loi religieuse, $35 \%$ des textes étant adaptés des coutumes et des traditions locales. D'ailleurs, un des avantages du Code suisse, qui a joué en faveur de ce choix, a été la grande place qu'il laisse aux juges, appelés à adapter cette matrice législative à la réalité sociale locale. Produit composite du féminisme d'État, ce code civil reflète l'esprit transitoire d'une époque : c'est précisément au moment où le multiculturalisme de l'Empire disparaît et où les chrétiens quittent ces terres, que la laïcité est proclamée, et que la restriction du mariage aux membres d'une même religion est interdite.

\section{Le féminisme, un outil de modernisation de l'État}

19 Plusieurs chercheurs insistent sur le fait que la femme turque a accédé à la citoyenneté grâce aux initiatives politiques de la classe dominante qui défendait un projet de modernisation précis. Dans ce cadre, Mustafa Kemal Atatürk aurait utilisé la qualité de citoyen offerte aux femmes turques dès 1934 comme une stratégie pour faire progresser l'occidentalisation du pays, transformant ainsi la femme turque en instrument de modernisation. Cependant, en définissant le féminisme d'État comme un statu quo socioculturel à l'intérieur duquel un État fort et centralisé met en place un projet de modernisation, Dicle Kogacioglu nous incite à voir, derrière les ruptures, les continuités qui persistent ${ }^{25}$. Selon elle, le féminisme d'État en Turquie a finalement soutenu un statu quo patriarcal, puisque, même après l'introduction des nouvelles lois, la famille reste plus importante que l'émancipation féminine : avant tout, les femmes restent pour la loi turque des épouses et des mères.

Comme nous l'avons vu, le leadership d'Atatürk et la création d'un "État père » ne doivent pas occulter l'importance des efforts accomplis par des générations de femmes turques, même si la période de leur action correspond à un "féminisme patriotique ". Mais cette continuité, qui définit le caractère interactif du féminisme d'État, ne doit pas non plus masquer le maintien des structures patriarcales, même quand une politique en faveur des femmes est menée. Ainsi, Deniz Kandiyoti, qui considère qu'Atatürk a émancipé les femmes turques sans les libérer, pose la question de savoir si son projet 
n'avait pas pour but de créer un État patriarcal moderne ${ }^{26}$. Toutefois, dans ce contexte transitoire, même la notion de modernité semble problématique (car liée aux puissances étrangères qui ont causé la chute de l'Empire). L'idéologue turquiste Ziya Gökalp et la Société turque d'histoire, à partir du début des années 1930, ont soutenu que la société préislamique turque était égalitaire, et les Turcs anciens, démocrates et féministes. Selon cette rhétorique qui fait du passé un réservoir de valeurs où il faut puiser pour construire le futur, il ne fallait pas regarder vers l'Occident, mais vers son propre passé pour réformer la société.

Malgré ce type d'argumentation, le projet modernisateur d'Atatürk a souvent été assimilé à une forme de " colonialisme interne " permettant à une élite dirigeante de définir sa mission comme civilisatrice face à «des forces traditionnelles, des forces d'anti-progrès, donc anhistoriques, et vouées à la disparition ${ }^{27}$ ». Le féminisme d'État s'inscrit ainsi dans un projet de civilisation qui vise à créer "l'homme nouveau ", même si «"l'homme nouveau" du réformisme kémaliste sera plutôt une femme ${ }^{28}$ ".

Jenny White nous rappelle que « le bras bureaucratique et civilisateur de la République était trop faible pour accéder aux coins reculés de la Turquie", même si le gouvernement y a envoyé des maîtresses d'école et des professeures, souvent des jeunes femmes d'origine rurale, qui devaient représenter et modeler les normes culturelles du nouvel État ${ }^{29}$. Même la rhétorique kémaliste qui exalte le passé des anciens Turcs (qui auraient été des féministes et des démocrates avant l'heure) n'a pu résoudre cette contradiction interne à tout projet de modernisation qui marque le passage d'un mode de vie traditionnel et rural à une culture urbaine et porteuse des valeurs progressistes. En essayant de diminuer le fossé entre la ville et la campagne, ces initiatives ont nécessairement été marquées par une tension entre modernité et tradition $^{30}$. Comme le constate Bruno Latour :

Lorsque les mots « moderne », « modernisation », « modernité » apparaissent, nous définissons par contraste un passé archaïque et stable. [...] « Moderne » est donc asymétrique par deux fois: il désigne une brisure dans le passage régulier du temps; il désigne un combat dans lequel il y a des vainqueurs et des vaincus ${ }^{31}$.

Si la Guerre de libération a donné la victoire aux Turcs, les Grecs, qui s'étaient investis de la mission de porter les lumières de la civilisation en Orient après la création de l'État grec en 1830, ont eu du mal à accepter la modernité des réformes kémalistes. Dans un discours prononcé en 1923, une féministe grecque, Athina Gaïtanou-Gianniou analyse ainsi les rapports entre la femme et la politique :

La préparation de la femme pour la vie politique ne se fera pas d'en haut, avec l'accord des hommes qui trouveront cinq-six femmes capables d'exercer de hautes responsabilités qu'ils exhiberont à d'autres pays avec un snobisme national comme Kemal l'a fait avec sa fameuse "hanim» ministre (perifimi hanoumissa ypourgina) -, mais elle se fera d'en bas ${ }^{32}$.

$\mathrm{Si}$ les féministes grecques regardent avec suspicion l'émancipation des leurs homologues turques, dès qu'elles obtiennent le droit de vote aux élections municipales en 1930 (sous condition de savoir lire et écrire et d'avoir plus de $30 \mathrm{ans}^{33}$ ), elles sont confrontées à une série de problèmes qui dépassent la sphère du féminisme stricto sensu et qui les font entrer dans celle de la modernisation de l'État grec. Étant donné que les femmes grecques qui remplissent les critères doivent s'inscrire sur les listes électorales, les associations féministes se mobilisent pour leur expliquer comment faire, car :

- pour prouver leur identité, elles doivent présenter la carte d'électeur de leur père ou de leur mari ; 
- puisque jusqu'en 1910 les registres de naissance sont mal tenus en Grèce, pour prouver leur âge, il faut le témoignage du prêtre de leur paroisse ou de deux témoins, ou l'acte de baptême ; en l'absence de ces documents, «l'appréciation de l'âge » qu'effectuera l'employé de la mairie qui est chargé de leur dossier suffira ;

- pour prouver leur scolarisation, il faut le bulletin de l'école ou un document certifié par le directeur de l'école ${ }^{34}$.

En effet, le droit de vote aux élections municipales fait entrer, pour la première fois, les femmes grecques dans les registres de l'État:

Faites le nécessaire pour obtenir le plus rapidement votre carte d'électeur [...] pour améliorer vos conditions de vie, la santé de vos enfants, le confort de votre foyer, mais aussi parce que le fait d'avoir un certificat fiable et sous la main de votre identité vous sera utile dans de nombreuses occasions : vous n'aurez plus besoin de chercher des témoins ou de faire des dépenses inutiles ${ }^{35}$.

Les papiers d'identité sont considérés, dans ce cadre, comme un progrès destiné à faciliter la vie des citoyennes-électrices qui étaient impliquées jusque-là dans les modes d'identification traditionnels et relationnels que sont le témoignage, l'attestation et la connaissance interpersonnelle. Le passage de la preuve testimoniale (qui implique le besoin de recourir à des témoins pour prouver son identité) à la preuve écrite produite par les services de l'État s'avérera non seulement irréversible, mais aussi à l'origine des "sociétés de surveillance" du xxI ${ }^{\mathrm{e}}$ siècle. Comme le dit Gérard Noiriel, "le nouveau système d'identification à distance doit impérativement s'assurer que l'identité réelle des personnes correspond bien à leur identité légale ${ }^{36}$ ». Dans les années 1930, c'est la satisfaction d'une revendication féministe centrale qui accéléra l'établissement des modèles d'identification bureaucratiques et la création du premier état civil « mixte "

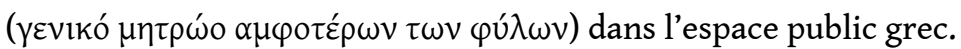

\section{Les années 1980, la « seconde vague » du mouvement féministe grec et turc}

Si dans les années 1920-1930, le féminisme en Grèce et en Turquie était lié à la modernisation de l'État, deux nouveaux paramètres le caractérisent dans les années 1980. D'une part, nous avons l'influence de la seconde vague du féminisme des années 1960-1970 qui, avec sa fameuse devise "le privé est politique ", a politisé des questions qui apparaissaient jusqu'alors comme relevant de la sphère privée (violence familiale, contraception, avortement), tout en considérant dans le même temps les normes juridiques comme des piliers des institutions patriarcales à combattre. D'autre part, il faut souligner l'effort fait par ces pays pour se conformer aux directives de la Communauté européenne - afin de faire avancer le processus d'entrée pour la Turquie, et comme conséquence de son intégration en 1981 pour la Grèce. Cela pose la question, encore une fois, des relations internationales et de leur impact sur le féminisme d'État ${ }^{37}$.

Le mouvement des féministes turques émerge de nouveau dans les années 1980 . La première manifestation, qui mobilise environ 3000 femmes, est organisée par le mouvement féministe à Istanbul en mai 1987 contre les violences domestiques. En 1990, à Istanbul et, en 1991, à Ankara, sont fondés des centres d'accueil et d'aide aux femmes victimes de violences ${ }^{38}$. En 1998 , la Loi sur la protection de la famille est votée, sous la pression du mouvement féministe ${ }^{39}$. 
29 Si les féministes grecques et turques restent à distance dans les années 1920-1930 (années marquées par la confrontation armée entre les deux pays), les contacts se développent dans les années 1980, et ce malgré les événements dramatiques de Chypre en 1974. La presse féministe grecque publie souvent des textes concernant le mouvement féministe en Turquie. Ainsi, une revue qui est publiée à Thessalonique, Katina, mentionne les manifestations des femmes turques à Istanbul contre la violence domestique et critique la nomination de Semra Özal (la femme du Premier ministre) en tant que « Mère de l'année $1987^{40}$ ». Une autre revue féministe, Dini, publie un article assez élaboré, où il est aussi question de la politique de Turgut Özal et de son effort de garder sous contrôle le mouvement féministe: selon les féministes turques interviewées, son intérêt pour la santé des femmes et la contraception ne constitue qu'une tentative de consolider l'institution de la famille que le féminisme menacerait ${ }^{41}$.

30 Cette période a aussi été marquée en Turquie par un "nettoyage législatif». Ainsi, l'article 159 du Code civil, qui exigeait l'accord du mari pour que l'épouse puisse travailler en dehors de la maison, est supprimé, la Cour constitutionnelle le jugeant en contradiction avec l'article 10 de la Constitution qui prévoit l'égalité des sexes devant la $\operatorname{loi}^{42}$. L'article 153 concernant le maintien du nom de jeune fille est révisé en mai 1997; cependant, même après cette révision, les femmes turques ne peuvent garder leur nom de jeune fille que si elles utilisent simultanément le nom de leur mari ${ }^{43}$. Des révisions sont aussi apportées au Code pénal qui définissait jusque-là des peines différentes selon le statut de la femme: mariée ou célibataire, vierge ou pas ${ }^{44}$. Les articles 440 et 441 concernant l'adultère (qui était plus facile à prouver contre la femme) sont supprimés.

31 Les années 1980 ont été également décisives pour les Grecques. Avec l'élection d'Andréas Papandréou en 1981, le terme "féminisme d'État» est introduit dans la réalité sociale et politique grecque pour la première fois. Son épouse, Margarita Papandréou, une féministe américaine de la seconde vague, devient la présidente de l'Union des Femmes de Grèce (EGE). Créée en 1976, cette organisation est affiliée au parti socialiste (PASOK) qu'Andréas Papandréou avait fondé le 3 septembre 1974.

32 Adoptée après la chute de la dictature des colonels (1967-1974), la Constitution de 1975 reconnaît pour la première fois l'égalité des droits et des obligations entre femmes et hommes, ainsi que l'attribution d'un salaire égal à travail égal. Après l'entrée de la Grèce dans la Communauté européenne en 1981, une série de mesures est prise pour améliorer la législation et supprimer les discriminations dont les femmes souffraient. Le Code civil est réformé en 1983 : entre autres, l'homme n'est plus le chef de famille (suppression de l'article 1387) et la décision de son épouse de travailler ne dépend plus de son accord ; de même, la femme peut garder son nom de jeune fille (suppression de l'article 1388). En mai 1986, l'avortement est légalisé (loi 1609) ${ }^{45}$.

33 En 1985, le poste de «Secrétariat général pour l'égalité des sexes» est créé; Hrysanthi Laïou-Antoniou, une membre de l'EGE, est la première à l'occuper. Celle-ci est souvent qualifiée par les féministes radicales grecques de "technocrate " qui présente toutes les mesures prises en faveur des femmes comme l'œuvre de l'EGE et du parti socialiste au pouvoir, en effaçant les années de lutte des différentes mouvances féministes grecques. C'est dans ce contexte que le terme «féminisme d'État» devient de plus en plus récurrent: il est utilisé par les féministes radicales grecques pour dénoncer la volonté du Parti socialiste de garder sous son contrôle le mouvement féministe ${ }^{46}$. 

des militantes, et tend à se les accaparer. En outre, il peut susciter un espoir illusoire de résolution de tous les problèmes des femmes, quelle que soit leur nature, grâce à l'intervention de l'État. Il pose également la question de l'obéissance et de la conformité aux lois, contrairement aux dynamiques du mouvement social et de la société civile qui présupposent l'adhésion et la participation active. Mais, dans certains, cas, comme nous le verrons maintenant, le féminisme d'État et le mouvement social ne sont pas des réalités distinctes, mais les pôles d'un continuum.

\section{Mouvement social et féminisme d'État}

Winpeace (Women's Initiative for Peace) a été créé par Margarita Papandréou et Zeynep Oral, en 1996, après un incident militaire entre Grecs et Turcs concernant le statut de leurs frontières dans la mer Égée. Cette ONG, qui œuvre pour la paix et la collaboration entre la Grèce, la Turquie et Chypre, se présente comme une tentative pour, d'une part, introduire une "approche féminine» dans les relations internationales, et, d'autre part, mobiliser les femmes «dans des activités de la "politique d'en bas" ». Winpeace bénéficiait en 2007 de financements de l'État (qui sont devenus difficiles à obtenir pour le côté grec avec l'arrivée de la droite au pouvoir en 2004), de l'Union européenne et d'institutions privées ${ }^{47}$.

En 2007, la présidente, du côté grec, était Fotini Sianou, la première femme à avoir assumé des responsabilités de haut niveau dans les instances syndicalistes grecques. La plupart des membres grecs de Winpeace étaient affiliés au Parti socialiste (PASOK). Margarita Papandréou, l'une des fondatrices du mouvement, est liée à une importante famille politique grecque, comme nous l'avons vu: ex-femme d'Andréas Papandréou, elle est aussi la mère de Giorgos Papandréou, qui était président du PASOK au moment de mes enquêtes. Certaines des militantes grecques de Winpeace ont occupé des postes à haute responsabilité, comme Hrysanthi Laïou-Antoniou, Première secrétaire pour l'égalité des sexes en Grèce, et une autre informatrice qui a longtemps travaillé pour les Nations Unies dans des programmes de développement pour les pays du Tiers Monde. Du côté turc, plusieurs militantes de Winpeace participent à l'association KADER qui a été créée en 1997 dans le but de promouvoir une représentation paritaire des femmes turques dans la vie politique. Certaines, comme Sirin Tekeli, ont été persécutées par le régime militaire de 1980 pour leur appartenance à des mouvements de gauche et leur activisme féministe.

Les militantes grecques se rappellent avec nostalgie la période où l'Union des femmes de Grèce (EGE) avait des milliers d'adhérentes dans tout le pays. Lors de cet "âge d'or ", 150 centres d'agrotourisme ont été créés, grâce au soutien de la Secrétaire générale pour l'égalité des sexes et les financements de l'Union européenne. Selon Eleni Papagaroufali et Eugenia Georges :

Pratiquement toutes les villageoises, quel que soit leur âge, ont été membres de ces coopératives, et ont développé un style de vie plus ou moins orienté vers l'égalité avec les hommes et l'émancipation, à travers, par exemple, la participation à des séminaires, des voyages en ville et même à l'étranger pour les affaires des coopératives $^{48}$.

Winpeace a mis en place un projet de ce type, pour la première fois en Turquie, dans trois villages de la péninsule de Karaburun à partir de 2002. Ayant joué un rôle 
important dans le développement de l'agrotourisme en Grèce, Hrysanthi Laïou-Antoniou a amorcé ce programme de Winpeace afin de faciliter sa transplantation en Turquie. Dans ce cas, nous avons le transfert d'un répertoire d'action du cadre étatique au champ militant et, simultanément, d'un pays à un autre : développée dans le cadre du féminisme d'État grec, cette initiative devient par la suite l'action phare d'une ONG qui essaie d'appliquer la même idée dans un pays voisin.

Winpeace est impliquée dans une «politique d'expertise» qui transforme ces militantes en nouvelles "spécialistes ", dont le positionnement par rapport à l'État varie selon les circonstances: quand le parti politique au pouvoir soutient les idées promulguées par Winpeace, des alliances se mettent en place, des financements sont accordés et ses membres accèdent à des postes importants ${ }^{49}$. Depuis la guerre en Irak, Winpeace étend son action vers le monde arabe. Pour ne donner qu'un seul exemple, Fotini Sianou a animé en 2007 un atelier de Conflict Resolution à Istanbul, dans le cadre d'un programme des Nations Unies (United Nations Population Fund, UNFPA), auquel participaient des femmes de différentes tribus iraquiennes. En effet, ces femmes sont devenues des "experts" qui exportent leur savoir-faire et sont sollicitées comme médiatrices dans d'autres conflits. Ces «spécialistes» issues de la société civile introduisent de nouvelles catégorisations dans la division du travail et brouillent les frontières entre l'expertise, le militantisme et sa bureaucratisation. Grâce à leur multipositionnalité, elles contribuent également à féminiser la vie publique, alternant une rhétorique d'égalité et une rhétorique de spécificité féminine.

Le cas de Winpeace fait apparaître les canaux de communication qui lient, à l'intérieur d'un continuum, le féminisme d'État et certaines mouvances féministes. Il montre aussi que le féminisme d'État d'Atatürk dans les années 1920 ne doit pas se mettre sur le même plan que le féminisme d'État de Papandréou dans les années 1980 : le premier a conduit à l'effondrement de l'activité protestataire féminine pendant, au moins, quatre décennies; le second, en revanche, a dû, tout au long, prendre en compte et dialoguer avec des militantes grecques de différentes mouvances. C'est peut-être pour cette raison qu'une féministe comme Sirin Tekeli, qui a forgé le terme "féminisme d'État » sur un ton critique à l'égard des réformes kémalistes, participe à un mouvement comme Winpeace et collabore avec les représentantes grecques du féminisme d'État des années 1980.

Tant en Grèce qu'en Turquie, les valeurs communautaires et nationales étaient encore dominantes dans les années 1920, alors que les deux pays restaient majoritairement agricoles. Définie par une urbanisation grandissante, la décennie de 1980 marque un tournant vers un individualisme plus prononcé. Dans ce contexte, ce sont des cas particuliers qui mobilisent et qui, une fois devenus emblématiques, rendent les discriminations envers les femmes encore plus visibles ${ }^{50}$. Enfin, c'est parce que le féminisme d'État est moins paternaliste dans les deux pays depuis les années 1990 que des groupes "pragmatiques" comme Winpeace, qui ne considèrent pas leur implication dans les rouages administratifs comme un compromis ou comme une perte d'autonomie, peuvent fonctionner comme des ponts entre la sphère étatique, la sphère partisane et les mouvements des femmes. 


\section{BIBLIOGRAPHIE}

ARAT Yeşim, 2006, “Women's challenge to citizenship in Turkey”, in Thalia DRAGONAS and Faruk BIRTEK (eds.), Citizenship and the Nation-State in Greece and Turkey, London: Routledge, pp. 203-224.

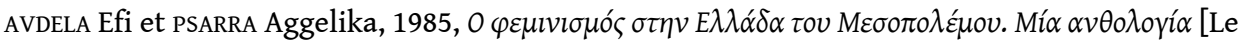

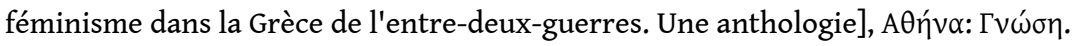

BASBUGU-YARAMAN Aysegül, 1996, «La femme turque dans son parcours émancipatoire (de l'empire ottoman à la république) », Cahiers d'Études sur la Méditerranée Orientale et le Monde Turco-Iranien, $\mathrm{n}^{\circ}$ 21, mis en ligne le 4 mai 2006. URL : http://cemoti.revues.org/556 (consulté le 3 mars 2016).

BASKAN Birol, 2014, From Religious Empires to Secular States: State Secularization in Turkey, Iran, and Russia, London-New York: Routledge.

BERENI Laure, 2007, « Du MLF au Mouvement pour la parité. La genèse d'une nouvelle cause dans l'espace de la cause des femmes ", Politix, $\mathrm{n}^{\circ}$ 78-2, p. 107-132. URL : http://www.cairn.info/ article.php?ID_ARTICLE=POX_078_0107 (consulté le 3 mars 2016).

DAUPHIN Sandrine, 2006, «L'élaboration des politiques d'égalité ou les incertitudes du féminisme d'État : une comparaison France/Canada ", Cahiers du Genre, n 3, p. 95-116. URL : http:// www.cairn.info/revue-cahiers-du-genre-2006-3-page-95.htm (consulté le 3 mars 2016).

EKAL Berna, 2011, “Women's Shelters and Municipalities in Turkey: Between Solidarity and Benevolence", EchoGéo, n 16, mis en ligne le 4 juillet 2011. URL : http://echogeo.revues.org/ 12509 (consulté le 3 mars 2016).

ELBIR Halid Kemal, 1956, « La réforme d'un code civil adopté de l'étranger », Revue internationale de droit comparé, vol. 8, n 1 , p. 53-64. URL : www.persee.fr/doc/ridc_0035-3337_1956_num_8_1_9659 (consulté le 3 mars 2016).

FIRAT Bilge, 2006, Dissident, but hegemonic: a critical review of feminist studies on gendered nationalism in Turkey, Thesis, State University of New York.

GöLE Nilüfer, 1994, «La revendication démocratique de l'islam », in Stéphane YERASIMOS (dir.), les Turcs. Orient et Occident, islam et laïcité, Paris : Autrement, p. 126-137.

KANDIYOTI Deniz A., 1987, “Emancipated but Unliberated? Reflections on the Turkish Case”, Feminist Studies, vol. 13, no. 2, pp. 317-338.

КоС̧АК Cemil, 2005, "Parliament Membership during the Single-Party System in Turkey (1925-1945)", European Journal of Turkish Studies, no. 3, mis en ligne le 4 mars 2015. URL : https:// ejts.revues.org/497 (consulté le 3 mars 2016).

KOGACIOGLU Dicle, 2006, “Citizenship in context: Rethinking women's relationships to the law in Turkey”, in Thalia DRAGONAS and Faruk BIRTEK (eds.), Citizenship and the Nation-State in Greece and Turkey, London: Routledge, pp. 273-301.

KONDYLI Mariana et PSARRA Aggelika, 1989, « Politique, genre, politique des femmes », Dini, revue féministe, $\mathrm{n}^{\circ} 4, \mathrm{p} .13-18$.

LAFON Jacques, 2001, Itinéraires. De l'histoire du droit à la diplomatie culturelle et à l'histoire coloniale, Paris : Publications de la Sorbonne. 
LATOUR Bruno, 1991, Nous n'avons jamais été modernes. Essai d'anthropologie symétrique, Paris : La Découverte.

LIBAL Kathryn, 2008, “Staging Turkish Women's Emancipation: Istanbul, 1935”, Journal of Middle East Women's Studies, vol. 4, no. 1, p. 31-52.

NOIRIEL Gérard, 2007, «Introduction », in Gérard NOIRIEL (dir.), l'identification. Genèse d'un travail d'État, Paris : Belin, p. 3-26.

PAPAGAROUfALI Eleni et GEORGES Eugenia, 1993, "Greek Women in the Europe of 1992: Brokers of European Cargoes and the Logic of the West", in George E. MARcus (dir.), Perilous States. Conversations on Culture, Politics, and Nation, Chicago-London: University of Chicago Press, pp. 235-254.

SERAÏDARI Katerina, 2008, « Du mouvement féministe au mouvement pacifiste. Winpeace, une organisation de militantes grecques, chypriotes et turques ", Nouvelles Questions Féministes, $\mathrm{n}^{\circ}$ 27-3, p. 57-71. URL : https://www.cairn.info/revue-nouvelles-questions-feministes-2008-3page-57.htm (consulté le 3 mars 2016).

SIRMAN Nürkhet, 2007, "Constituting the Modern Family as the Social in the Transition from Empire to Nation-State", in Caglar KEYDER and Anna FRAGOUDAKI, Ways to Modernity in Greece and Turkey. Encounters with Europe, 1850-1950, London-New York: I.B. Tauris, pp. 265-288.

TEKELI Sirin, 1994, «Les femmes, vecteur de la modernisation », in Stéphane YERASIMOS (dir.), les Turcs. Orient et Occident, islam et laïcité, Paris : Autrement, p. 138-152.

TEKELI Sirin, 1996, in « Table ronde I et II », Cahiers d'Études sur la Méditerranée Orientale et le Monde Turco-Iranien, $\mathrm{n}^{\circ}$ 21, mis en ligne le 4 mai 2006. URL : http://cemoti.revues.org/559 (consulté le 23 mars 2016).

TEKELI Sirin, 1996, « Les femmes républicaines et la place de la femme turque dans la société d'aujourd'hui : statut juridique et politique », Cahiers d'Études sur la Méditerranée Orientale et le Monde Turco-Iranien, $\mathrm{n}^{\circ}$ 21, mis en ligne le 4 mai 2016. URL : http://cemoti.revues.org/557 (consulté le 29 février 2016).

WHITE Jenny B., 2003, "State Feminism, Modernization, and the Turkish Republican Woman", NWSA Journal, vol. 15, no. 3, pp. 145-159.

\section{NOTES}

1. Très diverses, les mouvances féministes turques sont actuellement liées au socialisme, à l'islamisme, au kémalisme, à la gauche radicale et à des causes politiques comme l'autonomie kurde.

2. Sandrine DAUPHIN, 2006, «L'élaboration des politiques d'égalité ou les incertitudes du féminisme d'État : une comparaison France/Canada ", Cahiers du Genre, n 3, p. 110-112. URL : http://www.cairn.info/revue-cahiers-du-genre-2006-3-page-95.htm (consulté le 3 mars 2016).

3. Kathryn LIBAL, 2008, “Staging Turkish Women's Emancipation: Istanbul, 1935”, Journal of Middle East Women's Studies, vol. 4, no. 1, p. 36.

4. Ibid., p. 38. 
5. Aysegül BASBUGU-YARAMAN, 1996, «La femme turque dans son parcours émancipatoire (de l'empire ottoman à la république) », Cahiers d'Études sur la Méditerranée Orientale et le Monde Turco-Iranien, $\mathrm{n}^{\circ} 21, \S 72$, mis en ligne le 4 mai 2006. URL: http:// cemoti.revues.org/556 (consulté le 3 mars 2016).

6. Concernant l'évolution du féminisme en Turquie, Bilge Firat distingue quatre périodes : a) le "féminisme patriotique », selon le terme de Deniz Kandiyoti, qui s'étend de 1908 à la décennie de 1920 ; b) le "féminisme d'État», selon la définition de Sirin Tekeli, qui se prolonge jusque dans les années 1970 ; c) le « féminisme socialiste " des années 1970 ; et d) le « féminisme réel » à partir des années 1980. Voir Bilge FIRAT, 2006, Dissident, but hegemonic: a critical review of feminist studies on gendered nationalism in Turkey, Thesis, State University of New York, p. 94.

7. Nilüfer GöLE, 1994, «La revendication démocratique de l'islam», in Stéphane YERASIMOS (dir.), les Turcs. Orient et Occident, islam et laïcité, Paris : Autrement, p. 130.

8. Jacques LAFON, 2001, Itinéraires. De l'histoire du droit à la diplomatie culturelle et à l'histoire coloniale, Paris : Publications de la Sorbonne.

9. Ibid., p. 103.

10. Ibid., p. 103, note 1 .

11. Sirin TEKELI, 1996, « Les femmes républicaines et la place de la femme turque dans la société d'aujourd'hui : statut juridique et politique », Cahiers d'Études sur la Méditerranée Orientale et le Monde Turco-Iranien, $\mathrm{n}^{\circ} 21$, $\$ 6$, mis en ligne le 4 mai 2016. URL : http:// cemoti.revues.org/557 (consulté le 29 février 2016).

12. Birol BASKAN, 2014, From Religious Empires to Secular States: State Secularization in Turkey, Iran, and Russia, London-New York: Routledge, p. 69.

13. Voir Nükhet SIRMAN, 2007, "Constituting the Modern Family as the Social in the Transition from Empire to Nation-State", in Caglar KEYDER and Anna FAGOUKADI, Ways to Modernity in Greece and Turkey. Encounters with Europe, 1850-1950, London-New York: I.B. Tauris, p. 281. Sirman, qui soutient que les réformes kémalistes en faveur des femmes sont liées non seulement à la transition de l'Empire à l'État-nation, mais aussi au passage de la famille étendue à la famille nucléaire, cite une figure majeure du mouvement féministe, Nezihe Muhittin, qui déclarait en 1924 que les enfants élevés dans le cadre de la polygamie ne sont pas patriotes.

14. Halid Kemal ELBIR, 1956, «La réforme d'un code civil adopté de l'étranger », Revue internationale de droit comparé, vol. 8, $\mathrm{n}^{\circ} 1$, p.58. URL: www.persee.fr/doc/ ridc_0035-3337_1956_num_8_1_9659 (consulté le 3 mars 2016).

15. Sirin TEKELI, 1996, «Les femmes républicaines et la place de la femme turque dans la société d'aujourd'hui : statut juridique et politique », op. cit., § 13.

16. Kathryn LIBAL, 2008, "Staging Turkish Women's Emancipation: Istanbul, 1935", op. cit., p. 44.

17. Cemil кос̧ак, 2005, "Parliament Membership during the Single-Party System in Turkey (1925-1945)", European Journal of Turkish Studies, no. 3, § 4 et $\S 6$, mis en ligne le 4 mars 2015. URL : https://ejts.revues.org/497 (consulté le 3 mars 2016). 
18. Halid Kemal ELBIR, 1956, «La réforme d'un code civil adopté de l'étranger », op. cit., p. 58-59. URL : www.persee.fr/doc/ridc_0035-3337_1956_num_8_1_9659 (consulté le 3 mars 2016).

19. Dicle KOGACIOGLU, 2006, “Citizenship in context: Rethinking women's relationships to the law in Turkey", in Thalia DRAGONAS and Faruk BIRTEK (eds.), Citizenship and the Nation-State in Greece and Turkey, London: Routledge, p. 285.

20. Voir Sirin TEKELI, 1994, «Les femmes, vecteur de la modernisation", in Stéphane YERASIMOS (dir.), les Turcs. Orient et Occident, islam et laïcité, Paris : Autrement, p. 138-152. L'auteur (p.143) présente une enquête récente menée à Istanbul parmi des femmes, dont les résultats montrent comment certaines pratiques, valeurs et idées promulguées par les réformes kémalistes n'ont pas été intériorisées par l'ensemble de la population féminine.

21. Voir Dicle Kogacioglu, 2006, “Citizenship in context: Rethinking women's relationships to the law in Turkey", op. cit., p. 284 ; Voir aussi Sirin TEKELI, 1996, «Les femmes républicaines et la place de la femme turque dans la société d'aujourd'hui : statut juridique et politique », op. cit., §9.

22. Ibid., § 9.

23. Sirin TEKELI, 1996, in "Table ronde I et II", Cahiers d'Études sur la Méditerranée Orientale et le monde Turco-Iranien, $\mathrm{n}^{\circ} 21, \S 19$, mis en ligne le 4 mai 2006. URL: http:// cemoti.revues.org/559 (consulté le 23 mars 2016).

24. Jenny B. wHITE, 2003, "State Feminism, Modernization, and the Turkish Republican Woman”, NWSA Journal, vol. 15, no. 3, p. 151.

25. Dicle KOGACIOGLU, 2006, “Citizenship in context: Rethinking women's relationships to the law in Turkey", op. cit., pp. 281-283.

26. Deniz A. KANDIYOTI, 1987, "Emancipated but Unliberated? Reflections on the Turkish Case”, Feminist Studies, vol. 13, no. 2, pp. 317-338.

27. Nilüfer GöLE, 1994, « La revendication démocratique de l'islam », op. cit., p. 127-128.

28. Ibid., p. 129.

29. Jenny B. WHITE, 2003, "State Feminism, Modernization, and the Turkish Republican Woman", op. cit., p. 147.

30. Hati Çirpan représente également cette tendance : cette villageoise non seulement s'est distinguée par son action pendant la Guerre de libération, mais elle a aussi été élue chef de son village (muhtar) avant de devenir l'une des 18 femmes députées élues au Parlement turc en 1935 Voir Jenny B. wHITE, 2003, "State Feminism, Modernization, and the Turkish Republican Woman", op. cit., p. 151.

31. Bruno LATOUR, 1991, Nous n'avons jamais été modernes. Essai d'anthropologie symétrique, Paris : La Découverte, p. 20.

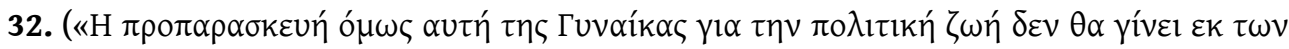

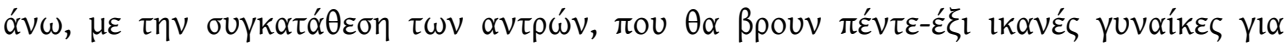

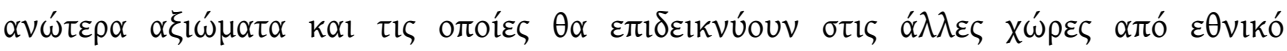

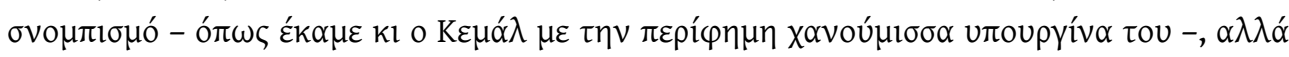

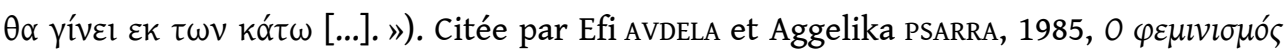

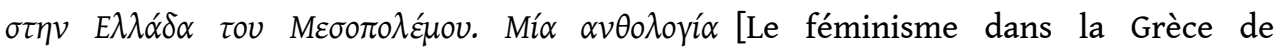

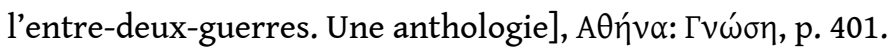


33. 240 femmes de Thessalonique ont été les premières femmes à voter en Grèce, lors des élections du 14 décembre 1930. À la fin de l'année 1931, seules 656 femmes ont été inscrites sur la liste électorale de Thessalonique. Il est considéré que seule $10 \%$ de la population féminine grecque remplissait à l'époque les critères requis, tandis que des Grecs hommes, illettrés, votaient sans problème à l'époque. Le droit au vote et à l'éligibilité au niveau national a été accordé aux Grecques en 1952.

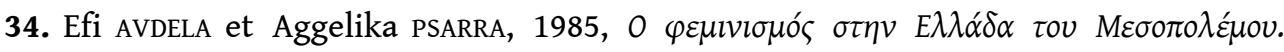
Mía $\alpha v \theta 0 \lambda$ oría [Le féminisme dans la Grèce de l'entre-deux-guerres. Une anthologie], op. cit., p. 373, 507-508, 511-512.

35. Ibid., p. 509.

36. Gérard NOIRIEL, 2007, "Introduction ", in Gérard NOIRIEL (dir.), l'identification. Genèse d'un travail d'État, Paris : Belin, p. 18.

37. Les conventions internationales entrent aussi dans cette catégorie, dans la mesure où elles constituent des engagements pour les États signataires. Ainsi, la Grèce a signé la Convention des Nations Unies pour l'élimination des discriminations à l'encontre des femmes (CEDAW) en 1982 et la Turquie en 1985. Comme le constate Laure Bereni, "l'ONU et la Communauté européenne sont des sites de production de nouveaux outils juridiques et de nouvelles rhétoriques en faveur de la cause des femmes en politique dès la décennie 1980 ». Voir Laure BERENI, «Du MLF au Mouvement pour la parité. La genèse d'une nouvelle cause dans l'espace de la cause des femmes ", Politix, $\mathrm{n}^{\circ} 78-2$, p. 125. URL : http://www.cairn.info/article.php?ID_ARTICLE=POX_078_0107 (consulté le 3 mars 2016).

38. Voir Berna EKAL, 2011, "Women's Shelters and Municipalities in Turkey: Between Solidarity and Benevolence", EchoGéo, $\mathrm{n}^{\circ}$ 16, mis en ligne le 4 juillet 2011. URL : http:// echogeo.revues.org/12509 (consulté le 3 mars 2016). Ekal analyse ces centres d'accueil comme des institutions bureaucratiques qui révèlent les tensions existantes entre le pouvoir central et le pouvoir local, mais aussi comme des lieux liés à la question patriarcale de l'honneur.

39. Yeşim ARAT, 2006, "Women's challenge to citizenship in Turkey", in Thalia DRAGONAS and Faruk BIRTEK (eds.), Citizenship and the Nation-State in Greece and Turkey, London: Routledge, p. 212.

40. Katina, $\mathrm{n}^{\circ}$ 2, décembre 1987, p. 50.

41. Dini, revue féministe, $\mathrm{n}^{\circ} 4$, juin 1989, p. 85-90.

42. Sirin TEKeLI, 1994, «Les femmes, vecteur de la modernisation », op. cit., p. 149.

43. Dicle KOGACIOGLU, 2006, "Citizenship in context: Rethinking women's relationships to the law in Turkey", op. cit., p. 297, note 12.

44. Ibid., p. 278.

45. À titre de comparaison, le droit à l'avortement a été légalisé en Turquie en 1983, sous condition de l'accord des deux conjoints pour les femmes mariées. En Grèce, la législation de l'époque ne distinguait pas les femmes selon leur statut marital, mais selon leur âge (adultes ou mineures) - ce qui a constitué un nouvel horizon de lutte pour les féministes, étant donné que les mineures devaient avoir l'accord de leurs parents pour avorter.

46. Voir, par exemple, Mariana KONDYLI et Aggelika PSARRA, 1989, "Politique, genre, politique des femmes ", Dini, revue féministe, $\mathrm{n}^{\circ} 4, \mathrm{p}$. 13-18. L'article met en parallèle la 
démission de Margarita Papandréou de la présidence de l'EGE et son divorce d'avec Andréas Papandréou ; Hrysanthi Laïou-Antoniou a aussi démissionné de son poste au même moment. La revue Katina ( ${ }^{\circ} 4$, mars 1989 , p. 7) critique également le « féminisme

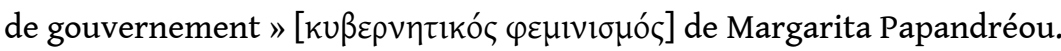

47. Ce terrain ethnographique a été effectué en novembre 2007. Pour une analyse des idées et des actions de cette ONG, voir Katerina SERAÏDARI, 2008, "Du mouvement féministe au mouvement pacifiste. Winpeace, une organisation de militantes grecques, chypriotes et turques", Nouvelles Questions Féministes, nº $27-3$, p. 57-71. URL : https:// www.cairn.info/revue-nouvelles-questions-feministes-2008-3-page-57.htm (consulté le 3 mars 2016).

48. Eleni papagaroufali et Eugenia Georges, 1993, "Greek Women in the Europe of 1992: Brokers of European Cargoes and the Logic of the West", in George E. MARcus (dir.), Perilous States. Conversations on Culture, Politics, and Nation, Chicago-London: University of Chicago Press, p. 252, note 16.

49. Comme cela s'est passé en Grèce avec certains membres de Winpeace, après le retour du Parti socialiste (PASOK) au pouvoir en automne 2009. Fotini Sianou a été nommée en janvier 2010 représentante de la Grèce aux Nations Unies pour les affaires concernant l'égalité des sexes. De même, Theodora Kokla a été nommée, en décembre 2009, Secrétaire générale de la préfecture de la Macédoine orientale et de Thrace (région qui abrite une population turque importante, exemptée de l'échange des populations selon le traité de Lausanne et toujours soumise à la loi religieuse) : elle est ainsi devenue la première femme à occuper ce poste.

50. Par exemple, l'article 438 du Code pénal turc stipulant que si la victime d'un violeur est une prostituée la peine est réduite de deux tiers, a été supprimé après une campagne très active des féministes qui se sont mobilisées à la suite du jugement d'un cas de viol commis à Antalya. Voir Sirin TEKELI, 1994, «Les femmes, vecteur de la modernisation », op. cit., p. 149.

\section{RÉSUMÉS}

Cet article examine les variations du fonctionnement du féminisme d'État dans deux pays «frères-ennemis ", la Turquie et la Grèce, pendant deux périodes charnières: les années 1920-1930, marquées par les réformes législatives kémalistes et la modernisation de l'État dans ces deux pays, et les années 1980 qui apportent des révisions au Code civil sous la pression $\mathrm{du}$ mouvement féministe. Ces juxtapositions montrent non seulement comment le droit constitue une construction sociale et politique malléable, mais aussi les rapports entre le féminisme d'État et le mouvement des femmes. Enfin, Winpeace, une ONG créée en 1996, qui rassemble des militantes grecques, turques et chypriotes, permet d'étendre cette analyse jusqu'aux années 2000 - décennie définie par l'émergence d'un militantisme féministe pragmatique et bureaucratisé.

This article examines the variations of function of state feminism in two enemy brothers, Turkey and Greece, during two pivotal periods: the 1920'-1930', which were marked by the Kemalist 
legislative reforms and the state modernization in the two countries, and the 1980', which brought reforms to the civil code under the pressure of the feminist movement. These juxtapositions show not only how law constitutes a malleable social and political construction, but also the relations between state feminism and women's movement. Winpeace, a NGO created in 1996, which gathers Greek, Turkish and Cypriot activists, allows extending the analysis to 2000 ' - a decade defined by the emergence of a pragmatic and bureaucratized feminist activism.

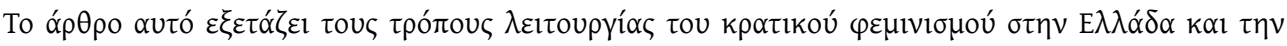

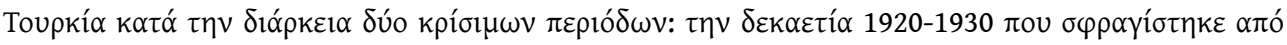

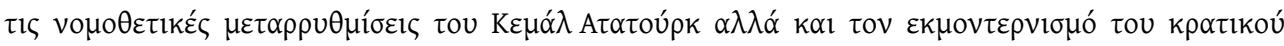

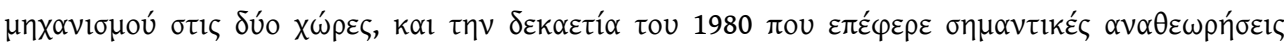

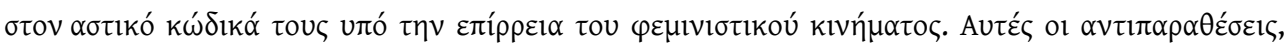

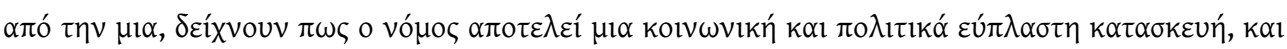

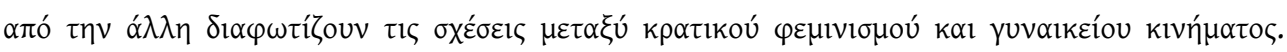

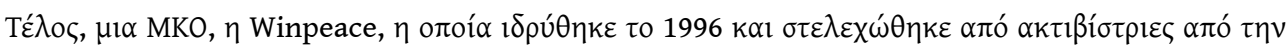

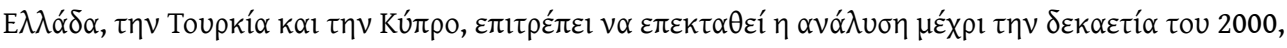

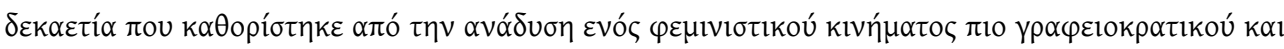

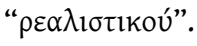

\section{INDEX}

motsclestr Devlet Feminizm, Toplumsal hareket, Mevzuat, Türkiye, Yunanistan, Yirminci yüzyıl, Sosyal tarih

motsclesmk ДРЖАВНИОТ ФЕМИНИЗМОТ, СОЦИЈАЛНО ДВИЖЕњЕ, ЗАКОНОДАВСТВО, ТУРЦИЈА, ГРЦИЈА, ДВАЕСЕТТИОТ ВЕК, СОЦИЈАЛНАТА ИСТОРИЈА

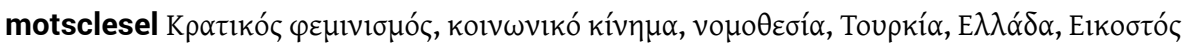

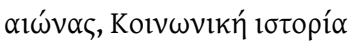

Thèmes : Histoire sociale

Mots-clés : féminisme d'État, féminisme d'État, mouvement social, mouvement social, législation, législation, Union des femmes de Grèce

Keywords : State feminism, social movement, legislation, Turkey, Greece, Twentieth century, Social history

Index géographique : Turquie, Grèce

Index chronologique : vingtième siècle

\section{AUTEUR}

\section{KATERINA SERAÏDARI}

Membre associée du LISST, Université Toulouse II-Le Mirail 